\title{
STATE-OF-THE-ART IN UAV REMOTE SENSING SURVEY - FIRST INSIGHTS INTO APPLICATIONS OF UAV SENSING SYSTEMS
}

\author{
Helge Aasen ${ }^{a^{*}}$

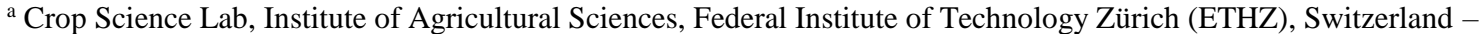 \\ helge.aasen@usys.ethz.ch
}

\author{
Commission I, WG I/1
}

KEY WORDS: spectral remote sensing, UAV, calibration, protocols, survey, platforms, sensors

\begin{abstract}
:
UAVs are increasingly adapted as remote sensing platforms. Together with specialized sensors, they become powerful sensing systems for environmental monitoring and surveying. Spectral data has great capabilities to the gather information about biophysical and biochemical properties. Still, capturing meaningful spectral data in a reproducible way is not trivial.

Since a couple of years small and lightweight spectral sensors, which can be carried on small flexible platforms, have become available. With their adaption in the community, the responsibility to ensure the quality of the data is increasingly shifted from specialized companies and agencies to individual researchers or research teams. Due to the complexity of the data acquisition of spectral data, this poses a challenge for the community and standardized protocols, metadata and best practice procedures are needed to make data intercomparable.

In November 2016, the ESSEM COST action Innovative optical Tools for proximal sensing of ecophysiological processes (OPTIMISE; http://optimise.dcs.aber.ac.uk/) held a workshop on best practices for UAV spectral sampling. The objective of this meeting was to trace the way from particle to pixel and identify influences on the data quality / reliability, to figure out how well we are currently doing with spectral sampling from UAVs and how we can improve. Additionally, a survey was designed to be distributed within the community to get an overview over the current practices and raise awareness for the topic. This talk will introduce the approach of the OPTIMISE community towards best practises in UAV spectral sampling and present first results of the survey (http://optimise.dcs.aber.ac.uk/uav-survey/).
\end{abstract}

This contribution briefly introduces the survey and gives some insights into the first results given by the interviewees.

\section{INTRODUCTION}

\subsection{Motivation}

The acquisition of geospatial data has transformed tremendously in the last decade. With the rise of UAVs as sensing platforms in combination with lightweight and small sensors, flexible sensing systems have become available for a wide audience. While this has enabled new ways of data acquisition (Aasen et al., 2015; Burkart et al., 2015; Honkavaara et al., 2016; Lucieer et al., 2014; Zarco-Tejada et al., 2012), this has also led to a democratisation of geospatial data acquisition in general (Aasen and Bolten, in review). But if more and more people are able to collect their own data, also knowledge about data acquisition and processing needs to be distributed among them. Besides, the question arises on how comparable data from different sensor and operated by different people, research groups or companies is.

Within the COST action on Innovative optical Tools for proximal sensing of ecophysiological processes (OPTIMISE, http://optimise.dcs.aber.ac.uk/), funded by the European Union, we explore the opportunities of UAVs to capture spectral data for ecological and physiological questions at multiple scales. One major effort is to establish best practice procedures for UAV spectral sampling (BUS). To assess the current state-ofthe-art of spectral remote sensing with UAVs we have designed a survey to ask you about your experiences on this topic and identify future needs to support the community. In this contribution, we will briefly introduce the survey a present some preliminary results.

\subsection{The survey}

The survey was designed with SurveyMonkey (SurveyMonkey, 2017) and can be found at http://optimise.dcs.aber.ac.uk/uavsurvey/. It consists of 51 questions that are grouped by different topics. Depending on previous answers, the following questions are filter such that only relevant questions are shown. This results in a time of 5 to 20 minutes to fill out the survey. The first questions ask about some information of the interviewee, their background and their experience with UAV remote sensing.

The next block of questions asks for details on the spectral UAV sensing system, in case the interviewee has its own. This block is relatively extensive since it is used to estimate the stateof-the-art of current spectral sensing systems. It includes questions about the sensor and its calibration, but also about the protocols used during the sensing procedure.

The next block of questions investigates the platforms that are currently used as well as information about the sensing scenario, such as flying height and speed. The last couple of questions give the opportunity to give more broad answers. These include the interviewees perspective on the biggest challenges and visions of UAV remote sensing.

The survey was opened in April 2017. So far, the survey has been published through various email lists, to the OPTIMISE community (http://optimise.dcs.aber.ac.uk) and at the 10th EARSeL SIG Imaging Spectroscopy Workshop (http://www.earsel.org/SIG/IS/workshops/10-IS-Workshop/). In the coming month it will circulated at further workshops and 
conferences of the remote sensing and earth observation community. Also other communities are welcome. Suggestions for conferences or e-mail lists are appreciated.

\section{PRELIMNARY RESULTS OF THE SURVEY}

\subsection{Current and planned use of UAVs}

Until now (Mid July 2017), 155 people have participated in the survey. Of those, more than the half have their own sensing system.

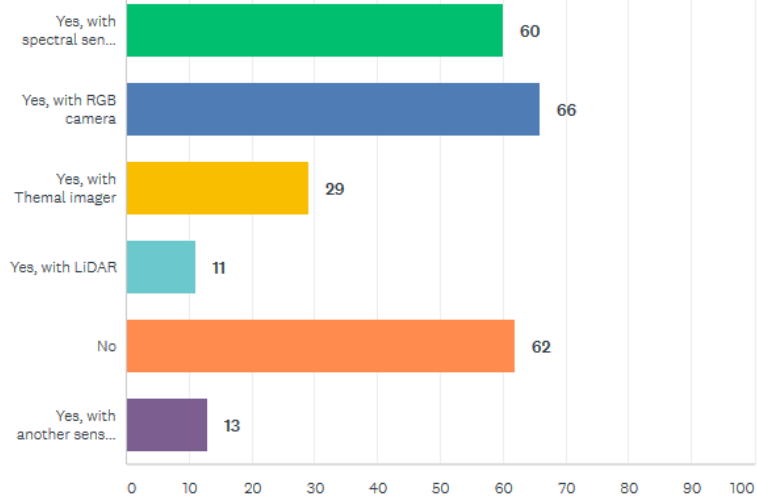

Figure 1 Results of the question: Do you have your own sensing system (sensor and UAV)? And if yes, what sensors do you deploy on it (multiple answers possible)?

Figure 1 shows the results of the question on if interviewees have their own sensing system and what sensor they deploy on them. about $40 \%$ percent have a sensing system with a spectral sensor, $45 \%$ with a RGB camera, $20 \%$ with a thermal imager, $7 \%$ with a LiDAR system and $8 \%$ with another sensor such as altimetry and sounder or magnetics sensor. Of those who have not yet a UAV sensing system, $1 / 3$ is planning to acquire a UAV in near future (Figure 2).

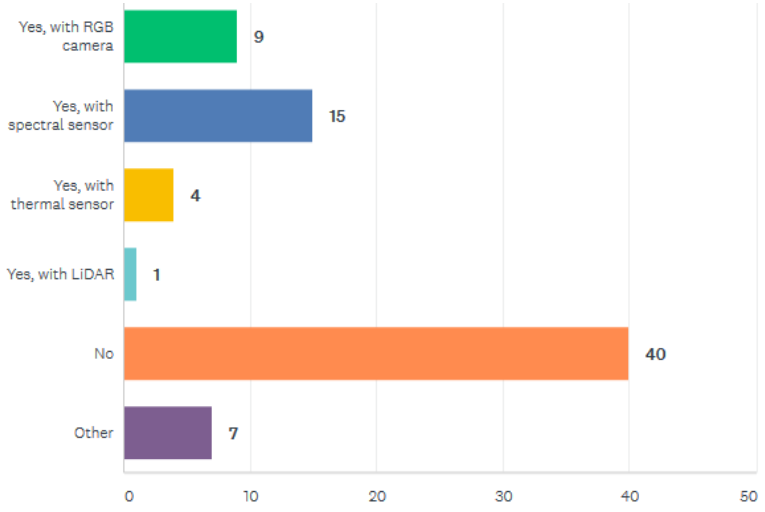

Figure 2 Are you planning to aquire a UAV in the near future?

\subsection{Users of UAV sensing systems}

The following results are based on the people that have already deployed UAV sensing systems. Most with a UAV sensing system come from Germany and Australia. In total people from 21 countries stated that they use of UAVs

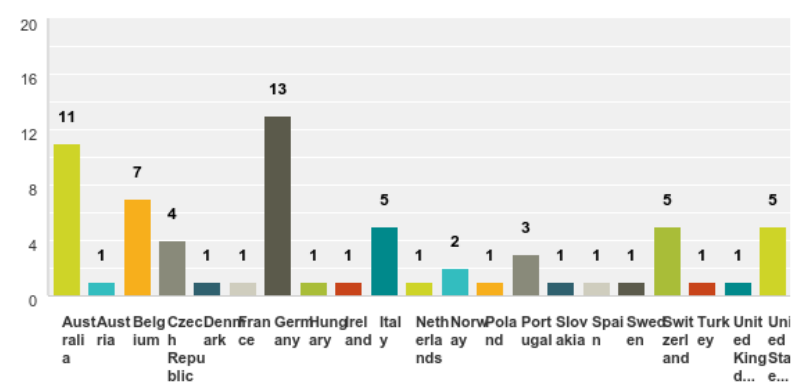

Figure 3 Distribution of participants that already use a UAV sensing system.

While the participants of a survey on the state-of-the-art on digital airborne photogrammetry in 2009 mostly were national mapping agencies (Honkavaara et al., 2009), so far most participants of this survey are researchers. In addition, UAV and software manufactures seem to have their own UAVs. Besides, also farmers have started to use UAVs (Figure 4).

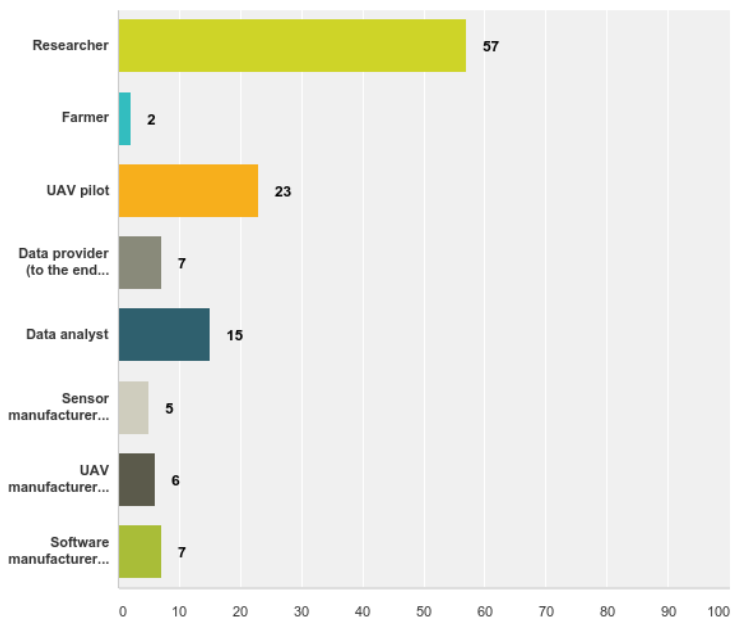

Figure 4 Current occupation of those who have an own UAV sensing system (multiple answers possible).

These people work in a wide range of fields. Most UAV users work in the field of agriculture, ecosystem monitoring and forests. Interestingly, also a large group of people are affiliated to sensor calibration and validation. This result could be biased by the group of participants that have fill out survey. Both the OPTIMISE and EARSeL IS community have a high share of experts in sensor calibration and validation activates. Also, sensor $\mathrm{cal} / \mathrm{val}$ might not me the primary interest of some of the people. With more and more people being able to set up their own spectral sensing system, more people also need to engage themselves with sensor cal/val activities (Aasen and Bolten, in review). 


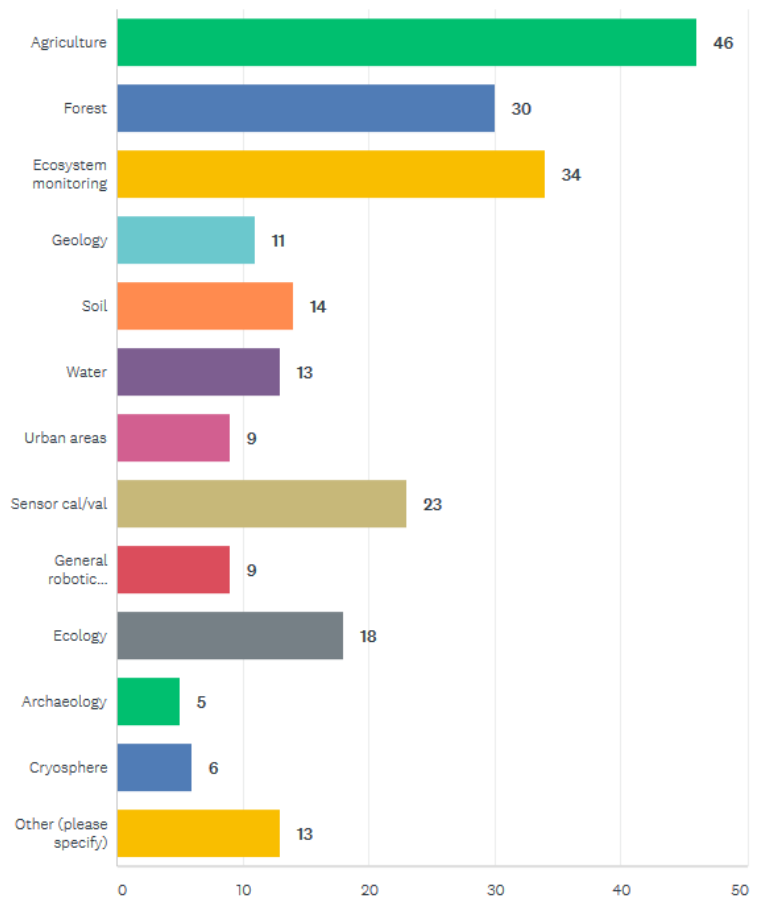

Figure 5 Fields of application of UAV sensing systems (multiple answers possible).

Generally, most people are rather new to field of UAV remote sensing. While there are a couple of people with more than 5 years of experience in UAV operation, most people have less than 3 years of experience (Figure 6).

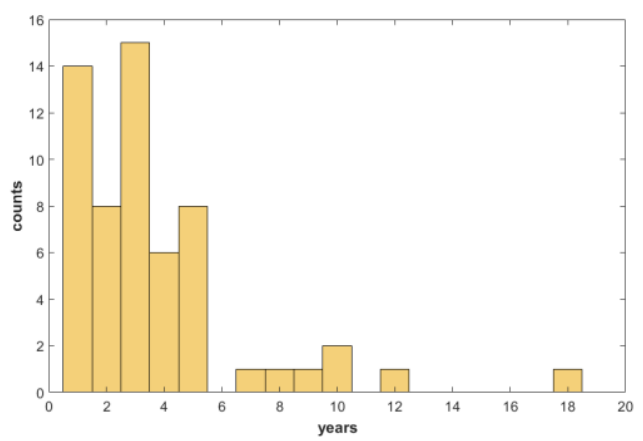

Figure 6 Experience in UAV operation in number of years of people with an own UAV sensing system.

\subsection{Usage of UAV sensing systems}

UAV sensing campaigns have been and are planned to be carried out in more than 17 countries. In line with the people who already have their sensing system in place (c.f. section 2.2), most campaigns are carried out in Germany and Australia. Besides, campaigns are carried out in many European countries, but also in countries beyond such as Jamaica, Namibia, China and India (Figure 7). At the end of the survey a more in depth analysis need to investigate, how many UAV teams carry out their research in foreign countries. This might also give some insight in how legislation issues affects UAV research.

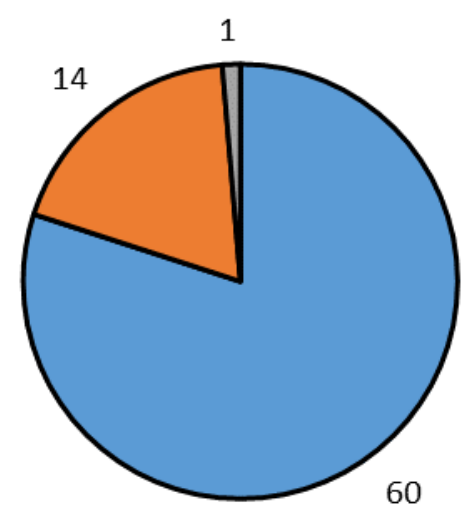

\section{口Multi-rotor $\boldsymbol{\square}$ Fixed-wing $\boldsymbol{\square}$ Idon't know}

Figure 8 Used types of UAV platform.

Figure 8 shows the type of platform that is used by the interviewees. Clearly, most people use Multi-rotor UAVs. Aligning with this, also rather low flying altitudes are used. Most campaigns are carried out below $100 \mathrm{~m}$, while it is most common to fly at around $50 \mathrm{~m}$. In one case, the typical flying altitude was state to be $2000 \mathrm{~m}$ (Figure 9). In this case, most likely a large fixed wing UAV is used.

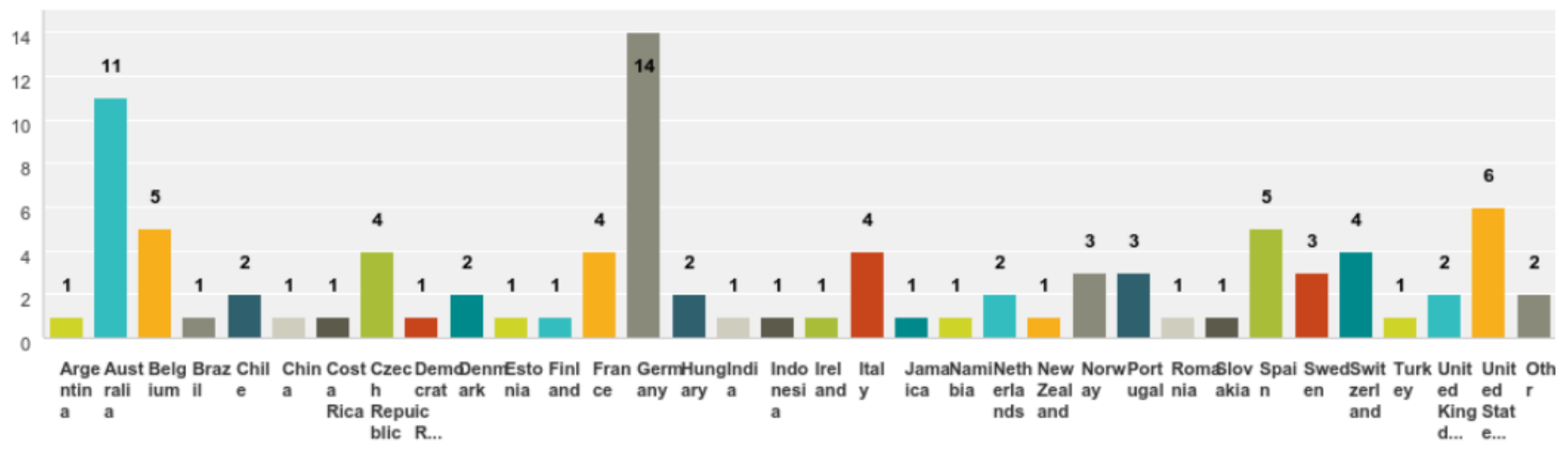

Figure 7 Countries were UAV campaigns are (planned to be) carried out. 


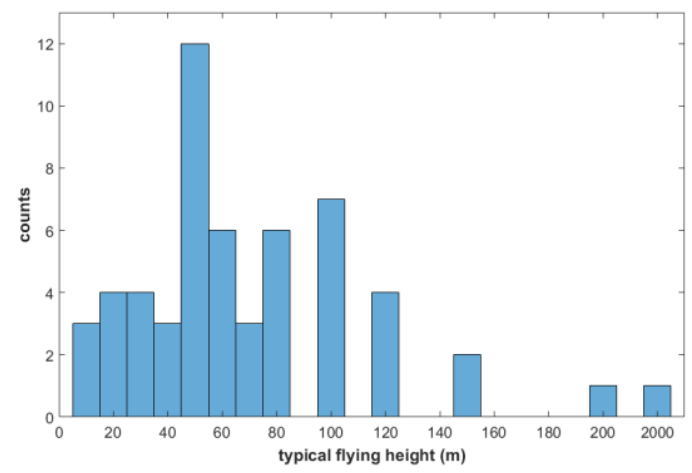

Figure 9 Typical flying height in meter (m) of UAV campaigns.

\section{CONCLUSIONS}

Although these results are just preliminary, they already show the diversity of UAV applications. Until now, only a few subcommunities have been addressed. In the coming months, hopefully more people will participate in the survey, which will give a useful insight into the activities currently carried out with UAVs.

\section{ACKNOWLEDGEMENTS}

We greatly acknowledge effort of the interviewees, who spend their valuable time to fill the survey. In addition, we are thankful for the contribution and work of the OPTIMSE community - in particular of those who participated in the OPTIMISE BUS workshop in Tartu (Andreas Burkart, Andreas Hueni, Antonis Kavvadias, Arko, Luccier, Dan Sporea, Eija Honkavaara, Enrico Tomelleri, Helge Aasen, Javier Pacheco, Joel Kuusk, Lea Hallik, Leonidas Toulios). Also, the funding by the European cooperation in science and technology for the ESSEM COST Action ES1309 Innovative optical Tools for proximal sensing of ecophysiological processes (OPTIMISE) is greatly acknowledged. Without its support and the input of the community, this survey would not have been possible.

\section{REFERENCES}

Aasen, H., Bolten, A., in review. Multi-temporal highresolution imaging spectroscopy with hyperspectral 2D imagers - from theory to application. RSE.

Aasen, H., Burkart, A., Bolten, A., Bareth, G., 2015. Generating 3D hyperspectral information with lightweight UAV snapshot cameras for vegetation monitoring: From camera calibration to quality assurance. ISPRS J. Photogramm. Remote Sens. 108, 245-259. doi:10.1016/j.isprsjprs.2015.08.002

Burkart, A., Aasen, H., Alonso, L., Menz, G., Bareth, G., Rascher, U., 2015. Angular Dependency of Hyperspectral Measurements over Wheat Characterized by a Novel UAV Based Goniometer. Remote Sens. 7, 725-746. doi:10.3390/rs70100725

Honkavaara, E., Arbiol, R., Markelin, L., Martinez, L., Cramer, M., Bovet, S., Chandelier, L., Ilves, R., Klonus, S., Marshal, P., Schläpfer, D., Tabor, M., Thom, C., Veje, N., 2009. Digital Airborne Photogrammetry-A New Tool for Quantitative Remote Sensing?-A State-of-the-Art Review On Radiometric Aspects of Digital Photogrammetric Images. Remote Sens. 1, 577-605. doi:10.3390/rs1030577
Honkavaara, E., Hakala, T., Nevalainen, O., Viljanen, N., Rosnell, T., Khoramshahi, E., Näsi, R., Oliveira, R., Tommaselli, A., 2016. Geometric and reflectance signature characterization of complex canopies using hyperspectral stereoscopic images from uav and terrestrial platforms. ISPRS Int. Arch. Photogramm. Remote Sens. Spat. Inf. Sci. XLI-B7, 77-82. doi:10.5194/isprs-archives-XLI-B7-77-2016

Lucieer, A., Malenovský, Z., Veness, T., Wallace, L., 2014. HyperUAS-Imaging Spectroscopy from a Multirotor Unmanned Aircraft System: HyperUAS-Imaging Spectroscopy from a Multirotor Unmanned. J. Field Robot. 31, 571-590. doi:10.1002/rob.21508

SurveyMonkey, 2017. SurveyMonkey: Free online survey software \& questionnaire tool [WWW Document]. Surv. Free Online Surv. Softw. Quest. Tool. URL https://www.surveymonkey.net/

Zarco-Tejada, P.J., González-Dugo, V., Berni, J.A.J., 2012. Fluorescence, temperature and narrow-band indices acquired from a UAV platform for water stress detection using a microhyperspectral imager and a thermal camera. Remote Sens. Environ. 117, 322-337. doi:10.1016/j.rse.2011.10.007 